\title{
The Metric Outside a Fixed Charged Body in the Covariant Theory of Gravitation
}

\author{
Sergey G. Fedosin \\ PO box 614088, Sviazeva str. 22-79, Perm, Russia \\ E-mail: intelli@list.ru
}

\begin{abstract}
The metric outside a charged body is calculated. As part of the given approach it is shown that the gravitational and electromagnetic fields are equally involved in the formation of the metric tensor components. And the contribution of fields in the metric is proportional to the energy of these fields. From equations for the metric it follows that the metric tensor components are determined up to two constants.
\end{abstract}

\section{Introduction}

In the theory of gravitation the metric is needed for correct representation of theoretical conclusions and experimental results. The concept of metric is most important in the general theory of relativity (GTR), in which the metric tensor field plays the role of the gravitational field. As a result, transition of GTR to the Newtonian theory of gravitation is always performed by expanding the metric tensor components to the principal expansion terms. For example, for the gravitational field potential we obtain the following expression: $\psi \approx \frac{1}{2}\left(c^{2} g_{00}-c^{2}\right)$, where $g_{00}$ is the time component of the metric tensor. No matter how small the field is, in GTR first we must find $g_{00}$, in order to evaluate then the potential $\psi$.

In contrast to this, in the covariant theory of gravitation (CTG) the metric in the weak field automatically tends to the metric of the Minkowski flat space, while the gravitational field equations do not change their form due to the covariant notation of equations. In CTG first we obtain the scalar potentials $\psi$ and $\varphi$ of the gravitational and electromagnetic fields, the vector field potentials, the tensors, corresponding to these fields, and only after that we calculate the spacetime metric outside the body [1].

\section{The solution of the equation for the metric}

The tensor equation for the metric, which was found in [2] from the principle of least action, taking into account the cosmological constant gauge, in mixed indices has the following form:

$$
R_{\alpha}^{\beta}-\frac{1}{4} R \delta_{\alpha}^{\beta}=-\frac{1}{2 c k}\left(B_{\alpha}^{\beta}+U_{\alpha}^{\beta}+W_{\alpha}^{\beta}+P_{\alpha}^{\beta}\right)
$$

Outside the body, where the mass and charge densities are equal to zero, the scalar curvature $R$ and the stress-energy tensors $B_{\alpha}^{\beta}$ of the acceleration field and $P_{\alpha}^{\beta}$ of the pressure field are also equal to zero. This is due to the fact that the acceleration field describes the motion of the body particles, and the pressure field is associated with the pressure of the body particles on each other. Both of these fields exist only in the volume of the body under consideration. The equality of the scalar curvature $R$ to zero is associated with the gauge condition of the cosmological constant.

In the right side of (1) there are two stress-energy tensors of the gravitational $U_{\alpha}^{\beta}$ and electromagnetic $W_{\alpha}^{\beta}$ fields 


$$
\begin{aligned}
& U_{\alpha}^{\beta}=\frac{c^{2}}{4 \pi G}\left(\Phi_{\kappa \alpha} \Phi^{\kappa \beta}-\frac{1}{4} \delta_{\alpha}^{\beta} \Phi_{\mu \nu} \Phi^{\mu \nu}\right) . \\
& W_{\alpha}^{\beta}=\varepsilon_{0} c^{2}\left(-F_{\kappa \alpha} F^{\kappa \beta}+\frac{1}{4} \delta_{\alpha}^{\beta} F_{\mu \nu} F^{\mu \nu}\right) .
\end{aligned}
$$

Here $G$ is the gravitational constant, $\varepsilon_{0}$ is the vacuum permittivity, $\Phi_{\mu v}$ and $F_{\mu v}$ are the gravitational and electromagnetic tensors, respectively, $\delta_{\alpha}{ }^{\beta}$ is the unit tensor or the Kronecker delta. We consider that the speed of light $c$ is the same for the propagation of electromagnetic and gravitational effects through the field. The stress-energy tensor of the gravitational field in the form of (2) was presented in [1].

With regard to (2) and (3), from (1) we obtain

$$
R_{\alpha}^{\beta}=-\frac{1}{2 c k}\left(U_{\alpha}^{\beta}+W_{\alpha}^{\beta}\right)
$$

According to (4), the Ricci tensor $R_{\alpha}^{\beta}$, associated with the spacetime curvature outside a single body, depends on the gravitational and electromagnetic field strengths of this body. Next, we will use the notation described in [3].

Suppose there is a body with a spherical shape and the mater distribution which is symmetrical relative to the center of the sphere. In the static case, the metric depends neither on time nor on the angles of the spherical reference frame. Then the metric at an arbitrary point around the body will depend only on the radial coordinate $r$ connecting this point with the center of the sphere. It is therefore convenient to use the spherical coordinates $x^{0}=c t, x^{1}=r, x^{2}=\theta, x^{3}=\phi$, associated with the Cartesian coordinates by relations: $x=r \sin \theta \cos \phi, \quad y=r \sin \theta \sin \phi$, $z=r \cos \theta$. The metric tensor will be found in the following form

$$
g_{\alpha k}=\left(\begin{array}{cccc}
B & 0 & 0 & 0 \\
0 & -K & 0 & 0 \\
0 & 0 & -E & 0 \\
0 & 0 & 0 & -E \sin ^{2} \theta
\end{array}\right),
$$

where $B, K, E$ are the functions only of the radial coordinate $r$.

Since $g_{\alpha k} g^{k \beta}=\delta_{\alpha}^{\beta}$, it is possible to find the metric tensor with contravariant indices

$$
\begin{aligned}
g^{k \beta}= & \left(\begin{array}{cccc}
\frac{1}{B} & 0 & 0 & 0 \\
0 & -\frac{1}{K} & 0 & 0 \\
0 & 0 & -\frac{1}{E} & 0 \\
0 & 0 & 0 & -\frac{1}{E \sin ^{2} \theta}
\end{array}\right) . \\
g_{33}= & -E \sin ^{2} \theta .
\end{aligned}
$$


The tensor of the gravitational field strength and the similar electromagnetic field tensor are expressed with the help of the corresponding 4-potentials with the covariant index [1]

$$
\begin{array}{ll}
\Phi_{r m}=\partial_{r} D_{m}-\partial_{m} D_{r}, & D_{m}=\left(\frac{\psi}{c},-\mathbf{D}\right)=\left(-\frac{G M}{r c}, 0,0,0\right), \\
F_{r m}=\partial_{r} A_{m}-\partial_{m} A_{r}, & A_{m}=\left(\frac{\varphi}{c},-\mathbf{A}\right)=\left(\frac{Q}{4 \pi \varepsilon_{0} r c}, 0,0,0\right),
\end{array}
$$

here $\psi$ is the scalar potential of the gravitational field around the massive body with the gravitational mass $M, \varphi$ is the scalar potential of the electromagnetic field around the body with the charge $Q$, the quantities $\mathbf{D}$ and $\mathbf{A}$ denote the vector potentials of the gravitational and electromagnetic fields.

We will assume that the vector potential $\mathbf{D}$ of the body is equal to zero, since that the body does not rotate, its particles move randomly in different directions and the vector potentials of particles compensate each other. Similarly, we assume that there are no directed electrical currents within the body, the magnetic moments of the substance particles are compensated and $\mathbf{A}=0$.

Using (7) and (2), we find the non-zero components $\Phi_{r m}$ and $U_{\alpha}^{\beta}$

$$
\begin{array}{cc}
\Phi_{01}=-\Phi_{10}=-\frac{G M}{r^{2} c}, & \Phi^{n s}=g^{n k} \Phi_{k m} g^{m s}, \\
U_{0}^{0}=U_{1}^{1}=-\frac{G M^{2}}{8 \pi B K r^{4}}, & U_{2}^{2}=U_{3}^{3}=\frac{G M^{2}}{8 \pi B K r^{4}} .
\end{array}
$$

From (8) and (3) we calculate the non-zero components $F_{r m}$ and $W_{\alpha}^{\beta}$

$$
\begin{gathered}
F_{01}=-F_{10}=\frac{Q}{4 \pi \varepsilon_{0} r^{2} c}, \quad F^{n s}=g^{n k} F_{k m} g^{m s}, \quad F^{01}=-F^{10}=-\frac{Q}{4 \pi \varepsilon_{0} B K r^{2} c}, \\
W_{0}^{0}=W_{1}^{1}=\frac{Q^{2}}{32 \pi^{2} \varepsilon_{0} B K r^{4}}, \quad W_{2}^{2}=W_{3}^{3}=-\frac{Q^{2}}{32 \pi^{2} \varepsilon_{0} B K r^{4}} .
\end{gathered}
$$

The Ricci tensor $R_{i k}$ is calculated according to the standard procedure using the Christoffel coefficients $\Gamma_{i k}^{s}$, i.e. the metric tensor and its derivatives

$$
\begin{aligned}
& \Gamma_{i k}^{s}=\frac{1}{2} g^{s m}\left(\partial_{i} g_{m k}+\partial_{k} g_{m i}-\partial_{m} g_{i k}\right) . \\
& R_{i k}=\partial_{s} \Gamma_{i k}^{s}-\partial_{k} \Gamma_{i s}^{s}+\Gamma_{i k}^{s} \Gamma_{s m}^{m}-\Gamma_{i m}^{s} \Gamma_{s k}^{m} .
\end{aligned}
$$

If we denote the derivatives with respect to $r$ by primes, then the non-zero Christoffel coefficients, expressed in terms of functions $B, K, E$ in the metric tensor (5) and (6), according to (11) are equal to 


$$
\begin{aligned}
& \Gamma_{01}^{0}=\Gamma_{10}^{0}=\frac{B^{\prime}}{2 B}, \quad \Gamma_{00}^{1}=\frac{B^{\prime}}{2 K}, \quad \Gamma_{11}^{1}=\frac{K^{\prime}}{2 K}, \quad \Gamma_{22}^{1}=-\frac{E^{\prime}}{2 K}, \quad \Gamma_{33}^{1}=-\frac{E^{\prime} \sin ^{2} \theta}{2 K}, \\
& \Gamma_{12}^{2}=\Gamma_{21}^{2}=\Gamma_{13}^{3}=\Gamma_{31}^{3}=\frac{E^{\prime}}{2 E}, \quad \Gamma_{33}^{2}=-\sin \theta \cos \theta, \quad \Gamma_{23}^{3}=\Gamma_{32}^{3}=\operatorname{ctg} \theta .
\end{aligned}
$$

Substituting (13) into (12), we find non-zero components of the Ricci tensor, and it also turns out that $R_{22}=R_{33}$. We obtain the expression for the Ricci tensor in mixed indices with the help of the metric tensor: $R_{\alpha}^{\beta}=R_{\alpha \mu} g^{\mu \beta}$. Using the obtained components $R_{\alpha}^{\beta}$ in the left side of (4), and the tensor components $U_{\alpha}{ }^{\beta}$ from (9) and $W_{\alpha}{ }^{\beta}$ from (10) in the right side (4), we find three independent equations

$$
\begin{aligned}
& B^{\prime \prime}-\frac{B^{\prime 2}}{2 B}-\frac{B^{\prime} K^{\prime}}{2 K}+\frac{B^{\prime} E^{\prime}}{E}=\frac{1}{8 \pi c k r^{4}}\left(G M^{2}-\frac{Q^{2}}{4 \pi \varepsilon_{0}}\right) . \\
& B^{\prime \prime}-\frac{B^{\prime 2}}{2 B}-\frac{B^{\prime} K^{\prime}}{2 K}+\frac{2 B E^{\prime \prime}}{E}-\frac{B E^{\prime 2}}{E^{2}}-\frac{B K^{\prime} E^{\prime}}{K E}=\frac{1}{8 \pi c k r^{4}}\left(G M^{2}-\frac{Q^{2}}{4 \pi \varepsilon_{0}}\right) . \\
& \frac{B E^{\prime \prime}}{E}+\frac{B^{\prime} E^{\prime}}{2 E}-\frac{B K^{\prime} E^{\prime}}{2 K E}-\frac{2 B K}{E}=-\frac{1}{8 \pi c k r^{4}}\left(G M^{2}-\frac{Q^{2}}{4 \pi \varepsilon_{0}}\right) .
\end{aligned}
$$

Equations (14) - (16) contain the second derivatives with respect to the current radius $r$, marked by two primes. If we subtract (15) from (14) and multiply the result by $\frac{E}{B E^{\prime}}$, it gives the following

$$
\begin{array}{ll}
-\frac{2 E^{\prime \prime}}{E^{\prime}}+\frac{E^{\prime}}{E}+\frac{K^{\prime}}{K}+\frac{B^{\prime}}{B}=0, & \frac{d}{d r}\left(\ln \frac{E K B}{\left(E^{\prime}\right)^{2}}\right)=0, \\
K=\frac{A_{1} E^{\prime 2}}{B E}, & \frac{K^{\prime}}{K}=\frac{2 E^{\prime \prime}}{E^{\prime}}-\frac{E^{\prime}}{E}-\frac{B^{\prime}}{B},
\end{array}
$$

where $A_{1}$ is a constant.

We will substitute in (16) the quantities $K$ and $\frac{K^{\prime}}{K}$ from (17)

$$
\frac{\left(B-4 A_{1}\right) E^{\prime 2}}{2 E^{2}}+\frac{B^{\prime} E^{\prime}}{E}=-\frac{1}{8 \pi c k r^{4}}\left(G M^{2}-\frac{Q^{2}}{4 \pi \varepsilon_{0}}\right) \text {. }
$$

Equation (18) is a quadratic equation for $E^{\prime}$ with the following solution

$$
E^{\prime}=\frac{-E B^{\prime} \pm E \sqrt{B^{\prime 2}-\frac{\left(B-4 A_{1}\right)}{4 \pi c k r^{4}}\left(G M^{2}-\frac{Q^{2}}{4 \pi \varepsilon_{0}}\right)}}{B-4 A_{1}}
$$


From (19) we can find the second derivative of $E$ with respect to $r$, that is $E^{\prime \prime}$. Substituting $E^{\prime}$ and $E^{\prime \prime}$ in (14) and also using (17) to substitute $\frac{K^{\prime}}{K}$ in (14), we obtain a differential equation of the second order for $B$

$$
B^{\prime \prime}+\frac{2 B^{\prime}}{r}-\frac{1}{8 \pi c k r^{4}}\left(G M^{2}-\frac{Q^{2}}{4 \pi \varepsilon_{0}}\right)=0 \text {. }
$$

The solution of equation (20) is the following expression

$$
B=A_{2}+\frac{A_{3}}{r}+\frac{1}{16 \pi c k r^{2}}\left(G M^{2}-\frac{Q^{2}}{4 \pi \varepsilon_{0}}\right)
$$

where $A_{2}$ and $A_{3}$ are some constants.

At infinity, where the gravitational and electromagnetic fields are close to zero, the metric tensor (5) must have the form of a tensor for Minkowski spacetime in spherical coordinates, for which $B=K=1, E=r^{2}$. Therefore, in (21) $A_{2}=1$ and in (17) and (19) $A_{1}=\frac{1}{4}$. Substituting these coefficients and $B$ from (21) in (19), we find: $E=r^{2}$. With the known $B$ and $E$ from (17) we see that $K=\frac{1}{B}$. With this in mind, the required functions for the metric around a single body in the covariant theory of gravitation will equal

$$
\begin{array}{cc}
B=g_{00}=1+\frac{A_{3}}{r}+\frac{1}{16 \pi c k r^{2}}\left(G M^{2}-\frac{Q^{2}}{4 \pi \varepsilon_{0}}\right), & E=-g_{22}=r^{2}, \\
K=-g_{11}=\frac{1}{B}=\frac{1}{1+\frac{A_{3}}{r}+\frac{1}{16 \pi c k r^{2}}\left(G M^{2}-\frac{Q^{2}}{4 \pi \varepsilon_{0}}\right)}, & g_{33}=-r^{2} \sin ^{2} \theta .
\end{array}
$$

\section{Conclusion}

In (22) the constants $A_{3}$ and $k$ are not determined from the equations for the metric, but the values of these constants can be found by comparison with the experimental data. According to (22), the contribution of the body charge $Q$ into the metric is opposite to the contribution of the gravitational mass $M$.

For comparison, we will use the Reissner-Nordström metric [4-5] for a charged body in the general theory of relativity (GTR)

$$
g_{00}=1-\frac{2 G M}{r c^{2}}+\frac{G Q^{2}}{4 \pi \varepsilon_{0} r^{2} c^{4}}, \quad g_{11}=-\frac{1}{1-\frac{2 G M}{r c^{2}}+\frac{G Q^{2}}{4 \pi \varepsilon_{0} r^{2} c^{4}}}, \quad g_{22}=-r^{2}, \quad g_{33}=-r^{2} \sin ^{2} \theta
$$

Expressions (23) in GTR do not contain indefinite coefficients, and in $g_{00}$ the term, containing $r^{2} c^{4}$ in the denominator, depends only on the charge $Q$. This reflects the absence of understanding of gravitation as an independent field in GTR, so in GTR there is no stress-energy 
tensor of the gravitational field and this tensor is not used in determining the metric in equation (1). As a result, the metric outside even uncharged body in GTR depends on the body and its mass in some mysterious way. Indeed, if there is no gravitational field, and nevertheless the body changes the metric near itself, there should be a mechanism that changes the metric and transfers the body's influence to any given point in space. This mechanism is not yet represented in GTR.

In the metric (22) of the covariant theory of gravitation, both gravitational and electromagnetic fields are equally represented in $g_{00}$ in the term, containing $r^{2}$ in the denominator. Both fields are the reason due to which the metric around the body is changed.

Let us suppose now that the constants $A_{3}$ and $k$ depend on the system's parameters as follows

$$
\begin{aligned}
& A_{3}=\frac{\alpha R_{b}}{M c^{2}}\left(\frac{G M^{2}}{R_{b}}-\frac{Q^{2}}{4 \pi \varepsilon_{0} R_{b}}\right)=-\frac{\alpha R_{b}}{M c^{2}}\left(c_{1} E_{g}+c_{2} E_{e}\right), \\
& \frac{1}{16 \pi c k}=\frac{\beta R_{b}}{M^{2} c^{4}}\left(\frac{G M^{2}}{R_{b}}-\frac{Q^{2}}{4 \pi \varepsilon_{0} R_{b}}\right)=\frac{\beta R_{b}}{M^{2} c^{4}}\left(c_{1} E_{g}+c_{2} E_{e}\right),
\end{aligned}
$$

where $R_{b}$ is the radius of the body, $E_{g}$ and $E_{e}$ denote the gravitational and electrical energies of the body's fields, the numerical coefficients $\alpha$ and $\beta$ should be determined further, the coefficients $c_{1}$ and $c_{2}$ depend on the mass and charge distribution, and in case of uniform density of the mass and charge of the body, are the same and are approximately equal to the value $5 / 3$.

In this case for $g_{00}$ from (22) we can write the following

$$
g_{00}=1-\frac{\alpha R_{b}}{M c^{2} r}\left(c_{1} E_{g}+c_{2} E_{e}\right)+\frac{\beta R_{b}^{2}}{M^{2} c^{4} r^{2}}\left(c_{1} E_{g}+c_{2} E_{e}\right)^{2}
$$

This relation can be interpreted so that in the static case the metric components $g_{00}$ and $g_{11}$ actually depend on the energy of the body's proper fields.

In our opinion a perspective theory, that allows us to explain from the physical standpoint the mechanism which transfers influence from some masses and charges to others at a distance by means of the field, is Fatio-Le Sage's theory of gravitation. In Fatio-Le Sage's model it is also possible to understand not only the interaction between the masses [6], but also between the electric charges [3]. Previously, we found the metric of the form (22) in [3], but now we considered the case of a charged body and took into account the contribution of the electric energy into the metric tensor components.

\section{REFERENCES}

[1] Fedosin S. G. Fizika i filosofiia podobiia ot preonov do metagalaktik. (Perm, 1999).

[2] Fedosin S.G. About the cosmological constant, acceleration field, pressure field and energy. vixra.org, 5 Mar 2014.

[3] Fedosin S. G. Fizicheskie teorii i beskonechnaia vlozhennost' materii (Perm, 2009).

[4] Reissner, H. (1916). Über die Eigengravitation des elektrischen Feldes nach der Einsteinschen Theorie. Annalen der Physik 50: 106-120. doi:10.1002/andp.19163550905.

[5] Nordström, G. (1918). On the Energy of the Gravitational Field in Einstein's Theory. Verhandl. Koninkl. Ned. Akad. Wetenschap., Afdel. Natuurk., Amsterdam 26: 1201-1208.

[6] Fedosin S.G. Model of Gravitational Interaction in the Concept of Gravitons. Journal of Vectorial Relativity, 2009, Vol. 4, No. 1, P.1 - 24. 